\title{
Índice de desempenho competitivo das empresas de polpa de frutas do Estado do Pará ${ }^{1}$
}

Antônio Cordeiro de Santana*

Resumo: O objetivo do trabalho foi construir um índice de desempenho competitivo para as empresas de polpa de frutas do Estado do Pará. Utilizaram-se as técnicas de análise fatorial e de regressão múltipla para estimar o IDC. Os resultados mostraram que, das 27 empresas analisadas, apenas uma empresa apresentou alto IDC e três IDC intermediários. Finalmente, observou-se uma relação positiva entre as variáveis margens de lucro e número de fornecedores e o IDC das empresas.

Palavras-chave: Índice de desempenho competitivo, frutas, Agroindústria, Amazônia.

Classificação JEL: L11 - Produção, preço e estrutura de mercado; Distribuição de tamanho das empresas.

Abstract: The objective of this paper was to construct a competitive performance index (CPI) for the fruit's pulp firms of State of Pará. A factor analysis and multiple regression models were used to estimate the CPI.

* D. Sc., Professor da Universidade Federal Rural da Amazônia (UFRA). O autor agradece as sugestões pertinentes de dois pareceristas anônimos da RER, que contribuíram para melhorar a compreensão e qualidade do texto. Erros e omissões remanescentes correm por conta do autor. santana@nautilus.com.br

${ }^{1} \mathrm{O}$ artigo é parte do relatório do projeto de pesquisa "Organização e competitividade das agroindústrias de polpa de frutas do Estado do Pará”, financiado pela FIDESA. 
Out the 27 firms analyzed, the results showed that only one firm presented a high CPI and three firms are obtaining intermediate CPI. Finally, there is a positive relationship between the firms' CPI and the variables profit's margins and number of supplier was observed.

Key words: Competitive performance index, fruit's pulp, agro-industry, Amazon.

Jel Classification: L11 - Production, Pricing, and Market Structure; Size Distribution of Firms

\section{Introdução}

O objetivo deste trabalho foi construir um índice de desempenho competitivo (IDC) para hierarquizar as empresas da indústria de polpa de frutas paraense, a partir dos dados de uma pesquisa de campo realizada em 27 empresas sediadas em 10 municípios de duas microrregiões do Estado do Pará: Metropolitana de Belém e Nordeste Paraense, no ano de 2003, e atualizada em 2004 (SANTANA et al., 2006).

A produção de frutas no Estado do Pará, sobretudo nas pequenas unidades de produção, está sendo desenvolvida em sistemas agroflorestais (SANTANA, 2004). Há um leque de 15 tipos de frutas tropicais (regionais e exóticas) sendo produzidas e beneficiadas pelas agroindústrias paraenses e em outros estados da Amazônia. As frutas exóticas de expressão são: abacaxi, acerola, maracujá e laranja. As frutas da Amazônia têm sabor diferenciado e as de maior destaque comercial são: açaí, cupuaçu, bacuri, taperebá e camu-camu, em que grande parte da produção ainda é extrativa. A produção de frutas manejada ou cultivada em sistemas agroflorestais, assim como o processamento agroindustrial é intensiva em mão-de-obra.

A produção de frutas gerou 626 empregos formais diretos e as agroindústrias geraram 1.374 empregos formais, em 2004, apenas nos municípios pesquisados, segundo informações do Registro Anual de Informações Sociais (RAIS) do Ministério de Trabalho. Em 2004, toda a cadeia produtiva de frutas do Estado do Pará (produção, processamento e distribuição) ocupou direta e indiretamente 106 mil pessoas 
(empregos formais e informais). O mercado nacional absorveu $50 \%$ da produção de polpa de frutas, o mercado estadual $41,5 \%$ e o mercado internacional $8,5 \%$ do total. O PIB estimado, neste mesmo ano, foi de R\$ 371,26 milhões (SANTANA et al., 2006).

O mercado internacional está no planejamento da maioria das agroindústrias entrevistadas, porém apenas um pequeno grupo dispõe de tecnologia para fabricar a polpa na qualidade e demais exigências deste mercado. A integração para frente das empresas com os distribuidores internacionais apenas se iniciou, pois não se domina a dinâmica desse mercado consumidor. Contudo, o sabor diferenciado ou "exótico" e o grande conteúdo energético, vitamínico e socioambiental das frutas regionais estão agradando ao consumidor estrangeiro, revelado pelo crescimento da demanda.

O valor das exportações de frutas, polpas e sucos de frutas do Estado do Pará foi de US\$33,94 milhões em 2005, com aumento de 45,73\% em relação a 2004. A receita com a exportação apenas do mix de polpa e suco de frutas foi de US $\$ 10,54$ milhões em 2005, incremento de $22,86 \%$ em relação a 2004. Do valor total das exportações do mix de polpa de frutas, realizado em 2005, a polpa de açaí representou 52,1\%.

A demanda do mix de polpa de frutas, além de superior, está evoluindo mais rápido do que a oferta (SANTANA, 2004; SANTANA et al., 2006). Concomitantemente, o mercado está exigindo qualidade, diversificação e diferenciação de produto, volume e regularidade no fluxo de produção e comercialização. Para atender a estas exigências, diante da elevada sazonalidade da produção, as empresas necessitam processar pelo menos 10 tipos de polpa de frutas diferentes. Esta lógica exige, portanto, plantas multiprodutos e expansão da oferta de frutas por meio de plantios racionais. O processo de ajuste na produção agroindustrial está em curso, porém de forma heterogênea e desarticulada.

O tecido empresarial está estruturado em microempresas $(51,9 \%$, com receita bruta até $\mathrm{R} \$ 244$ mil ou com até 19 empregados), pequenas empresas (40,7\%, com receita bruta entre $\mathrm{R} \$ 244$ mil e $\mathrm{R} \$ 1,20$ milhão ou com 20 a 99 empregados) e médias empresas $(7,4 \%$, com receita bruta entre $R$ \$ 1,20 milhão e $\mathrm{R} \$ 35$ milhões ou com 100 a 499 empregados), conforme estatuto da micro e pequena empresa (SANTANA et al. 2006). Há uma grande heterogeneidade no tecido empre- 
sarial no que tange à tecnologia, qualidade de produto, obtenção de informação, acesso a mercado e gestão empresarial. As médias empresas e algumas pequenas e microempresas estão trabalhando com um mix de 10 a 15 tipos de polpa de frutas congelada e/ou pasteurizada. Um pequeno grupo destas implantou as boas práticas e fabricação e está a caminho da certificação internacional por meio da Análise de Perigos e Pontos Críticos de Controle (APPCC). As microempresas, em geral, processam apenas um tipo de fruta, obrigando-as a fechar no período de entressafra.

O índice de concentração de mercado de Herfindahl-Hirschman (IHH) das agroindústrias, conforme Santana et al. (2006), foi de 1.130, revelando que o mercado é desconcentrado e as empresas operam em regime de concorrência monopolística, em função da diferenciação dos produtos em qualidade, embalagens, forma de distribuição e de gestão. A rivalidade entre as empresas é baixa, em função da grande demanda e da diferença de tamanho das plantas. Não há ameaça de produtos substitutos, em função do sabor exótico das frutas da Amazônia, do poder energético e teor de vitaminas, porém é real a ameaça de entrada de novas empresas de porte médio na indústria de polpa de frutas paraense. No momento, há três novas plantas em fase avançada da implantação. A interação das empresas com os fornecedores de frutas e com os clientes é embrionária. Porém, está em andamento o processo de organização e operação da integração dos elos de produção e processamento da cadeia produtiva de polpa de frutas.

Diante de um cenário de oportunidades de mercado nacional e internacional para os produtos regionais, não há estudos que possibilitem conhecer e orientar a trajetória de crescimento da indústria de polpa de frutas do Estado do Pará. A pesquisa coordenada por Santana et al. (2006), iniciada em 2003, foi o primeiro passo na direção do conhecimento sobre a organização da indústria de polpa de frutas, dimensão do mercado, estratégias competitivas e interação com os órgãos de fomento, de assistência técnica e instituições de ensino e pesquisa. Neste trabalho, apresenta-se o tópico relativo à construção de um indicador de desempenho competitivo, construído a partir de métodos científicos apropriados. 


\section{Metodologia}

\subsection{Fundamentação teórica}

Na literatura especializada em organização industrial existe um conjunto de elementos metodológicos que discute o conceito de competitividade, com base no índice de concentração e poder de mercado, economias de escala e de escopo, estratégias empresariais e confluência de forças sistêmicas que determinam o desempenho competitivo das empresas (BAIN, 1968; PORTER, 1990; COUTINHO; FERRAZ, 1994; ESSER et al., 1999; SHY, 1997; TIROLE, 2001).

Por competitividade empresarial entende-se o esforço realizado pelas empresas para continuarem no mercado, mantendo e/ou ampliando sua parcela de mercado. Este conceito de competitividade, definido com base nos postulados do modelo estrutura-conduta-desempenho (ECD) ou modelo tradicional da competitividade industrial proposto por Bain (1968), segundo Possas (1987), OCDE (1992), Coutinho e Ferraz (1994) e Farina e Zilbersztaja (1998), não é operacional. Na tentativa de superar essa dificuldade, Porter (1990) considerou que as variáveis que definem as dimensões do modelo ECD atuam de forma dinâmica e não estática como proposto. Na mesma direção a OCDE (1992), Coutinho e Ferraz (1994), Possas (1996) e Esser et al. (1999), propuseram que a competitividade empresarial é o resultado da interação sistêmica de conjuntos de fatores que podem ser agrupados nos níveis micro, macro, meso e meta, levando em conta os pontos relevantes das abordagens teóricas tradicionais e modernas (SANTANA, 2002).

Sinteticamente, o modelo ECD ou modelo tradicional postula que o desempenho competitivo da indústria é o resultado das relações de uma constelação de elementos organizados nas dimensões: Estrutura (concentração de fornecedores e clientes, diferenciação e diversificação de produtos, condição de entrada, formas de integração), Conduta (preços, estratégia de venda, pesquisa, desenvolvimento e inovação, tática geral) e Desempenho (alocação eficiente de recursos, margem de lucro, conservação de recursos, bem-estar social, emprego, parcela de mercado). Segundo este modelo, a estrutura determina a conduta e esta, isolada ou em conjunto com a estrutura, determinam a posição competitiva das 
empresas. Entretanto, os elementos do bom desempenho nem sempre são totalmente compatíveis entre si ou se relacionam completamente entre as dimensões, o que dificulta a criação de um índice preciso que possa ser adotado para avaliar as características de desempenho das empresas. Em busca de alternativa, Braga (1980) empregou métodos econométricos para determinar a estrutura de mercado e o desempenho da indústria brasileira, utilizando como variável dependente alguns dos indicadores de desempenho e como variáveis independentes um conjunto de variáveis indicadoras das dimensões estrutura e conduta. Apesar do rigor científico dos indicadores criados, sua aplicação esbarra nas limitações de estatísticas e na exigência de alto conhecimento para a estruturação dos modelos econométricos.

Porter (1990) propôs o Modelo de Cinco Forças (MCF) competitivas, mediante a reorganização dos elementos que influenciam a competitividade empresarial em cinco dimensões ou forças: poder do fornecedor (número de fornecedores, exigência de integração vertical, escassez de fornecimento), poder do cliente (número de clientes, concentração dos compradores, capacidade do setor, homogeneidade do setor), produtos substitutos (marca versus genérico, defasagem de valor-preço para outros produtos), entrantes potenciais (economia de escala, custo de capital elevado, falta de acesso a canais de distribuição, custo de transação elevado) e rivalidade empresarial (concentração do setor, taxa de crescimento, rigidez dos custos, tática de concorrência de preços). Porter entende que as vantagens competitivas devem ser construídas como um fenômeno sistêmico, determinado pela confluência da interação das empresas com seus fornecedores e clientes, visando atenuar as ameaças de empresas rivais, concorrentes potenciais e de produtos substitutos no mercado. Apesar do maior grau de operacionalidade deste modelo, dada a facilidade de obtenção de informações no âmbito operacional da empresa, não se tem um indicador que sumarize o resultado de tais efeitos. O padrão de análise disseminado por Porter $(1990,1999)$ e empregado em diversos estudos (FAIRBANKS; LINDSAY, 2002; KITABAYASHI, 2005; SILVA, 2006) se pauta na análise individual dos elementos de cada força e o vínculo é tecido mediante análise e alguns cruzamentos entre variáveis.

O modelo de competitividade sistêmica adiciona aos modelos anteriores a influência de elementos como infra-estrutura, política, cultura, 
inovação tecnológica e de gestão e o ambiente institucional que atuam em toda cadeia produtiva, além da dinâmica intertemporal do mercado (POSSAS, 1987; OCDE, 1992; COUTINHO; FERRAZ, 1994; FERRAZ et al., 1995; FARIAN; ZILBERSZTAJN,1998; ESSER et al., 1999; SANTANA, 2002). Assim, de acordo com Santana (2001), a abordagem sistêmica da competitividade pode ser considerada como uma paisagem que se move ao sabor da influência dos elementos agrupados nos ambientes de análise microeconômico (eficiência alocativa, capacidade de gestão, estratégias, inovação tecnológica, rede de integração), macroeconômico (política monetária, fiscal, cambial, orçamento), institucional (fatores sócio-culturais, governança, organização econômica, capacidade política) e de política setorial (infra-estrutura, educação, política ambiental e tecnológica, política regional). Pelo que se observa, a construção de um indicador de competitividade sistêmica torna-se ainda mais complexo. Em função disso, os estudos desenvolvidos por Coutinho e Ferraz (1994), Farina e Zilbersztajn (1998), Santana (2002) e Santana et al. (2005), utilizaram tabelas de freqüência para apoiar as análises de comportamento dos elementos de cada dimensão da competitividade sistêmica. Como no modelo de Porter, as interações entre as forças competitivas e a avaliação de conjunto se restringem, basicamente, ao campo das análises qualitativas e interpretativas. Portanto, existe uma lacuna no que se refere à construção de indicadores de desempenho competitivo capazes de refletir a dimensão do conceito de competitividade empresarial.

Neste trabalho, pretende-se adotar um método de maior rigor estatístico e que permite visualizar a confluência das diversas forças que influenciam a competitividade. Na indústria de polpa de frutas, é a primeira vez que se emprega a técnica da análise fatorial para construir um índice de desempenho competitivo para as empresas da indústria de polpa de frutas paraense.

Modelar estas dimensões não é tarefa fácil e constitui o desafio deste trabalho. A iniciativa foi estruturar um modelo estatístico para resumir a massa de informações em um conjunto reduzido de variáveis capaz de mais bem representar o movimento das empresas da indústria de polpa de frutas paraense na luta para assegurar seu espaço no mercado. As vantagens competitivas de curto prazo, geralmente, estão associadas aos diferenciais de custos resultantes das condições de fatores disponí- 
veis em dados territórios (no caso, disponibilidade de matéria-prima, mão-de-obra barata, crédito subsidiado etc.). Por outro lado, as vantagens competitivas sustentáveis ou de longo prazo se vinculam ao tamanho adequado da planta empresarial, treinamento contínuo de mão-deobra, qualidade, diversificação e diferenciação da produção, integração vertical e horizontal, visando agregar de valor a toda cadeia produtiva.

Acredita-se que as empresas da indústria de polpa de frutas paraense estão operando com grau de competitividade heterogêneo. A constatação científica da posição competitiva de cada empresa na indústria, porém, não é possível de ser identificada por meio da análise qualitativa por categoria de empresas, segundo o tamanho. Para evidenciar as empresas que estão operando com grau de competitividade alto, intermediário e baixo, construiu-se o IDC.

A construção do IDC foi realizada em três etapas. Na primeira etapa, empregou-se a técnica da análise fatorial para extrair fatores e estimar os escores fatoriais a serem usados no cálculo do IDC, que tem a finalidade de hierarquizar as empresas, conforme seu grau de desempenho competitivo. A segunda etapa consistiu em utilizar a proporção da variância explicada por cada fator em relação à variância total explicada pelo conjunto de fatores comuns, para determinar o peso associado a cada escore fatorial utilizado na construção do IDC. A última etapa consistiu em hierarquizar o IDC do maior para o menor valor obtido, visando revelar a posição competitiva de cada empresa na indústria de polpa de frutas das microrregiões Metropolitana de Belém e Nordeste Paraense do Estado do Pará. Adicionalmente, empregou-se um modelo econométrico para evidenciar a influência de algumas outras variáveis sobre o IDC das empresas, que ficaram de fora da análise fatorial.

\subsection{Modelo analítico}

A análise fatorial, de modo geral, é utilizada para analisar a estrutura das inter-relações (correlações) entre um grande número de variáveis, definindo um conjunto de dimensões latentes comuns que facilitam a compreensão da estrutura da nuvem de dados, chamados de fatores. Com o emprego dessa técnica, inicialmente pode-se identificar as dimensões isoladas da estrutura dos dados e então determinar o grau em que cada 
variável é explicada por cada dimensão ou fator. Depois dessa etapa, a análise fatorial pode ser empregada para reduzir a massa de dados.

Um modelo de análise fatorial pode ser apresentado na forma matricial como em Dillon e Goldstein (1984):

$$
X=\alpha F+\varepsilon
$$

Em que:

$\boldsymbol{X}=$ é o p-dimensional vetor transposto das variáveis observáveis, denotado por $\boldsymbol{X}=\left(x_{1}, x_{2}, \ldots, x_{p}\right)^{\prime}$;

$\boldsymbol{F}=$ é o q-dimensional vetor transposto de variáveis não-observáveis ou variáveis latentes chamadas de fatores comuns, denotado por, $\boldsymbol{F}$ $=\left(f_{1}, f_{2}, \ldots, f_{q}\right)^{\prime}$, sendo que $\mathrm{q}<\mathrm{p}$;

$\varepsilon=$ é o p-dimensional vetor transposto de variáveis aleatórias ou fatores únicos, $\varepsilon=\left(e_{1}, e_{2}, \ldots, e_{p}\right)^{\prime}$;

$\alpha=$ é a matriz $(\mathrm{p}, \mathrm{q})$ de constantes desconhecidas, chamadas de cargas fatoriais.

No modelo de análise fatorial pressupõe-se que os fatores específicos são ortogonais entre si e com todos os fatores comuns. Normalmente, $E(\varepsilon)=E(F)=0$ e $\operatorname{Cov}(\varepsilon, F)=0$.

A estrutura inicial utilizada para determinar a matriz de cargas fatoriais, em geral, pode não fornecer um padrão significativo de cargas das variáveis, por isso não é definitiva. A confirmação ou não dessa estrutura inicial pode ser feita por meio de vários métodos de rotação dos fatores (DILLON; GOLDSTEIN, 1984; JOHNSON; WICHERN, 1988). Nesta pesquisa, utilizou-se o método varimax de rotação ortogonal dos fatores. O método varimax é um processo em que os eixos de referência dos fatores são rotacionados em torno da origem até que alguma outra posição seja alcançada. O objetivo é redistribuir a variância dos primeiros fatores para os demais e atingir um padrão fatorial mais simples e teoricamente mais significativo (REIS, 2001; HAIR et al., 2005; SANTANA, 2005).

A escolha dos fatores foi realizada por meio da técnica de raiz latente. Esta técnica parte do princípio de que qualquer fator individual deve explicar a variância de pelo menos uma variável para que seja mantido para interpretação. Cada variável contribui com um valor 1 do autovalor total. Com efeito, apenas os fatores que têm raízes latentes ou autovalores maiores que 1 são considerados significantes e os demais 
fatores com autovalores menores do que 1 são considerados insignificantes e descartados (HAIR et al., 2005; MINGOTI, 2005; SANTANA, 2005). A matriz de cargas fatoriais, que medem a correlação entre os fatores comuns e as variáveis observáveis, é determinada por meio da matriz de correlação, conforme Dillon e Goldstein (1984).

Para a definição do Índice de Desempenho Competitivo (IDC), estimou-se a matriz de escores fatoriais após a rotação ortogonal da estrutura fatorial inicial. O escore fatorial, por definição, situa cada observação no espaço dos fatores comuns. Para cada fator $f_{j}$, o i-ésimo escore fatorial extraído é definido por $F_{i j}$, expresso da seguinte forma (DILLON; GOLDSTEIN, 1984; SPSS, 1997):

$$
F_{i j}=b_{1} x_{i 1}+b_{2} x_{i 2}+\ldots+b_{p} x_{i p} ; i=1,2, \ldots, n ; j=1,2, \ldots, p
$$

Em que:

$\boldsymbol{b}_{\boldsymbol{i}}$ são os coeficientes de regressão estimados para os $n$ escores fatoriais comuns;

$x_{i j}$ são as $n$ observações das $p$ variáveis observáveis.

A variável $F_{i j}$ não é observável, mas pode ser estimada por meio das técnicas de análise fatorial, utilizando-se a matriz de observações do vetor $x$ de variáveis observáveis. Em notação matricial, a equação 2, torna-se:

$$
F_{(n, q)}=x_{(n, p)} b_{(p, q)}
$$

Na equação $3, \boldsymbol{F}$ é a matriz da regressão estimada a partir dos $n$ escores fatoriais e que podem ser afetados tanto pela magnitude quanto pelas unidades de medida das variáveis $x$. Para contornar este tipo de problema, substitui-se a variável $x$ pela variável padronizada $w$, dada pela razão entre o desvio em torno da média e o desvio padrão de $\boldsymbol{x}$, como a seguir:

$$
w_{i j}=\frac{\left(x_{i}-\bar{x}\right)}{S_{x}}
$$

Com esses valores, modifica-se a equação 3 para gerar a equação 4 .

$$
F_{(n, q)}=\mathcal{W}_{(n, p)} \beta_{(p, q)}
$$


Na equação 4 , a matriz de pesos beta - $\beta$, com $q$ colunas e $p$ coeficientes de regressão padronizados, substitui $b$, dado que as variáveis estão padronizadas em ambos os lados da equação. Pré-multiplicando ambos os lados da equação 4 pelo valor $(1 / n) w$ ', em que n é o número de observações e $w$ ' é a matriz transposta de $w$, obtém-se:

$$
\frac{1}{n} w_{(p, n)}^{\prime} F_{(n, q)}=\frac{1}{n} w_{(p, n)}^{\prime} w_{(n, p)} \beta_{(p, q)}=R_{(p, p)} \beta_{(p, q)}
$$

A matriz $(1 / k) w^{\prime} w$ se constitui na matriz de variáveis inter-correlacionadas ou matriz de correlação entre as observações da matriz $x$, designada por $\boldsymbol{R}$. A matriz $(1 / \boldsymbol{k}) \boldsymbol{w}$ ' $\boldsymbol{F}$ representa a correlação entre os escores fatoriais e os próprios fatores, denotada por $\Lambda$. Reescrevendo a equação 5, tem-se que:

$$
\Lambda_{(p, q)}=R_{(p, p)} \beta_{(p, q)}
$$

Se a matriz $\boldsymbol{R}$ for não-singular, pode-se pré-multiplicar ambos os lados da equação 6 pela inversa de $\boldsymbol{R}$, obtendo-se:

$$
\beta=R^{-1} \Lambda
$$

Substituindo o vetor $\beta$ na equação 4, obtém-se o escore fatorial associado a cada observação, como a seguir:

$$
F_{(n, q)}=\mathcal{W}_{(n, p)} R_{(p, p)}^{-1} \Lambda_{(p, q)}
$$

O IDC FOI definido como uma combinação linear desses escores fatoriais e a proporção da variância explicada por cada fator em relação à variância comum. A expressão matemática é dada por:

$$
I D C_{i}=\sum_{j=1}^{q}\left(\frac{\lambda_{j}}{\sum_{j} \lambda_{j}} F P_{i j}\right)
$$

Em que $\lambda$ é a variância explicada por cada fator e $\Sigma \lambda$ é a soma total da variância explicada pelo conjunto de fatores comuns. $\mathrm{O}$ escore fatorial foi padronizado (FP) para se obter valores positivos dos escores originais e permitir a hierarquização das empresas, uma vez que os valores do IDC estão situados entre zero e um. A fórmula matemática proposta foi a seguinte: 


$$
I D C_{i}=\sum_{j=1}^{q}\left(\frac{\lambda_{j}}{\sum_{j} \lambda_{j}} F P_{i j}\right)
$$

Em que $F_{\min }$ e $F_{\max }$ são os valores máximo e mínimo observados para os escores fatoriais associados às empresas de polpa de frutas.

Para facilitar a interpretação dos resultados, foram estabelecidos os seguintes intervalos de valores do IDC, agrupando as empresas conforme seu grau de desempenho competitivo: valores do IDC igual ou superior a 0,70 são considerados altos; valores situados entre 0,35 e 0,69 são intermediários; valores inferiores a 0,35 são considerados baixos.

\subsubsection{Modelo econométrico}

Adicionalmente, procurou-se aferir a influência de outras variáveis não incluídas na análise fatorial sobre o IDC das empresas da indústria de polpa de frutas paraenses. Em função do tamanho da amostra e do grande número de variáveis necessárias relacionadas ao desempenho competitivo das empresas de polpa de frutas, buscou-se uma forma alternativa de aferir o grau de influência dessas variáveis sobre o IDC.

Para isso, empregou-se uma regressão múltipla. O modelo econométrico foi especificado da seguinte forma:

$$
I D C_{i}=a_{0}+a_{1} F N_{i}+a_{2} M_{i}+a_{3} C O_{i}+a_{4} O D P_{i}+e_{i}
$$

Em que:

$\boldsymbol{F N}_{i}$ é o número de fornecedores de matéria-prima (frutas) e outros insumos para as empresas processadoras de polpa de frutas. Espera-se obter uma relação positiva entre o número de fornecedores e o desempenho competitivo das empresas, dado que o custo de transação é baixo, vez que as empresas estão localizadas próximas da fonte de matéria-prima e os agricultores operam em regime de concorrência pura;

$M L_{i}$ é a margem de lucro das empresas de polpa de frutas, medida pela razão porcentual entre o lucro e o custo total de produção das empresas. Espera-se obter uma relação positiva entre a margem de lucro das empresas e seu desempenho competitivo, vez que este é um dos indicadores de desempenho competitivo; 
$\mathbf{C O}_{\boldsymbol{i}}$ é o nível de capacidade ociosa das empresas de polpa de frutas, medido em porcentagem da capacidade total instalada, dependendo das circunstâncias pode influenciar o desempenho competitivo das empresas mesmo em curto prazo;

$\boldsymbol{O D P _ { i }}$ é a organização em departamentos das empresas de polpa de frutas, contemplando departamento contábil, de pessoal, de vendas e marketing, por contribuir para racionalizar o processo de gestão empresarial, também influencia o desempenho competitivo das empresas;

$\boldsymbol{e}_{i}$ é o termo de erro aleatório, assumindo distribuição normal com média zero e variância constante.

Espera-se obter relação positiva entre essas variáveis e o IDC, exceto para a variável capacidade ociosa. O método de estimação utilizado foi o de mínimos quadrados ordinários. Analisou-se a influência de heterocedasticidade pelo método de White e de multicolinearidade pelo fator de variância inflacionária, conforme Santana (2003).

\subsection{Dados e variáveis}

Os dados utilizados na pesquisa são oriundos de pesquisa de campo, realizada junto às agroindústrias de polpa de frutas paraenses no ano de 2003 e algumas variáveis atualizadas em 2004 (SANTANA, et al., 2006).

As empresas entrevistadas foram eleitas com base nos cadastros do Serviço Brasileiro de Apoio às Micro e Pequenas Empresas (Sebrae) e da Delegacia Federal de Agricultura (DFA). Foram consideradas apenas as empresas formais e com seus produtos registrados no Ministério da Agricultura. Com base nesse critério, foram eleitos os 10 municípios das mesorregiões Metropolitana de Belém e Nordeste Paraense, onde essas empresas estão sediadas, para a efetivação da pesquisa. Em função do número reduzido de empresas, optou-se por entrevistar todas as empresas multiproduto (oito empresas) e, entre as empresas uniproduto, aquelas com possibilidade de fornecer informações para preencher a maioria das perguntas incluídas no questionário (19 empresas), perfazendo uma amostra de 27 empresas. O instrumento de coleta dos dados foi um questionário preparado para abranger todos os elementos de fundamento da análise de arranjos produtivos locais (REDESIST, 2004) e competitividade sistêmica (COUTINHO; FERRAZ, 1994; SANTANA, 2002). Neste artigo 
foi explorado apenas um bloco de questões orientadas para avaliar o desempenho competitivo das agroindústrias de polpa de frutas.

As variáveis incluídas na análise foram: quantidade de polpa de frutas de cada empresa, em toneladas (Quantidade); receita bruta obtida com a venda de polpa de fruta, em $\mathrm{R} \$$ (Receita); custo total de produção de polpa de frutas, em R\$ (Custo); taxa de concentração de mercado das empresas (TCM); número de empregados pelas empresas de polpa de frutas (Emprego); assistência técnica utilizada pelas empresas de polpa de frutas (A. Técnica); índice de inovação tecnológica empregado pelas empresas (Tecnologia); acesso a crédito de curto e de longo prazo por parte das empresas (Crédito) e indicador de diversificação das linhas de produção das empresas (Diversificação).

Nota-se que o número de variáveis é considerado grande em relação ao tamanho da amostra, que conta com apenas 27 empresas, numa ração de três observações para cada variável. A priori, o recomendado seria a utilização de pelo menos cinco observações para cada variável. Diante deste problema, foram feitos testes para aferir a adequação do método à amostra de dados. Os dois principais testes aplicados foram: teste de esfericidade de Bartlett, que avalia a significância geral da matriz de correlação, ou seja, testa a hipótese nula de que a matriz de correlação é uma matriz identidade; teste de Kaiser-Meyer-Olkin (KMO) se baseia no princípio de que a inversa da matriz de correlação se aproxima da matriz diagonal, para tanto compara as correlações entre as variáveis observáveis.

As fórmulas matemáticas destes testes são as seguintes (DILLON; GOLDSTEIN, 1984; REIS, 2001; MINGOTI, 2005):

$$
K M O=\frac{\sum_{i} \sum_{j} r_{i j}^{2}}{\sum_{i} \sum_{j} r_{i j}^{2}+\sum_{i} \sum_{j} a_{i j}^{2}}
$$

Em que $r_{i j}$ é o coeficiente de correlação da amostra entre as variáveis $x_{i}$ e $x_{j}$ e $a_{i j}$ é o coeficiente de correlação parcial entre as mesmas variáveis que é, simultaneamente, uma estimativa das correlações entre os fatores, eliminando o efeito das demais variáveis. Os $a_{i j}$ deverão assumir valores próximos de zero, uma vez que se pressupõe que os fatores são ortogonais entre si. Valores deste teste abaixo de 0,50 são inaceitáveis (HAIR et al., 2005). 
O teste de Bartlett de esfericidade testa a hipótese nula de que as variáveis são independentes, contra a hipótese alternativa de que as variáveis são correlacionadas entre si. Ou seja:

$$
\begin{aligned}
& \mathrm{H}_{0}: R=I \text { ou } \mathrm{H}_{0}: \lambda_{1}=\lambda_{2}=\ldots=\lambda_{\mathrm{p}}, \text { e é dado por: } \\
& \chi^{2}=-\left[n-1-\frac{1}{6}(2 p+5)\right] \cdot \ln |R| \text { ou } \\
& \chi^{2}=-\left[n-1-\frac{1}{6}(2 p+5)\right] \cdot \sum_{i=1}^{p} \ln \lambda_{i}
\end{aligned}
$$

Em que $|\boldsymbol{R}|$ é o determinante da matriz de correlação da amostra, $\lambda$ é a variância explicada por cada fator, $n$ é o número de observações e $p$ é o número de variáveis. A estatística tem uma distribuição assintótica de $\chi^{2}$ com $[0,5 . p \cdot(p-1)]$ graus de liberdade.

\section{Análise dos resultados}

Esta seção apresenta e discute os resultados gerados pelo modelo de análise fatorial, do índice de desempenho competitivo e da análise econométrica sobre as empresas de polpa de frutas paraenses.

\subsection{Análise fatorial}

A adequação da análise fatorial foi determinada pelos testes Bartlett e KMO. O teste de Bartlett avaliou a significância geral da matriz de correlação e apresentou um valor de 244,5 , indicando que as correlações, em geral, são significantes ao nível de $1 \%$ de probabilidade, ou seja, a matriz de correlação não é diagonal. O teste KMO, da ordem de 0,739 indicou que as variáveis estão correlacionadas e o modelo fatorial apresentou um nível bom de adequação aos dados.

Estes resultados respaldam o emprego da análise fatorial para a extração de fatores e a estimação dos escores fatoriais.

A matriz de correlação $R$, como descrito na metodologia, foi transformada por meio de um modelo fatorial para gerar a matriz de cargas fatoriais. As cargas das variáveis associadas aos fatores são interpretadas como identificadoras da estrutura latente das variáveis, que refletem as forças competitivas das empresas de polpa de frutas. O primeiro 
passo dessa tarefa reside em selecionar o número de componentes a serem mantidos para a estimação dos escores fatoriais.

A Tabela 1 contém os resultados da solução inicial e rotacionada para os nove fatores possíveis e seu relativo poder explanatório expresso pelos autovalores. Aplicando o critério da raiz latente, conforme Dillon e Goldstein (1984), três componentes foram mantidas ou extraídas. Observase que os três fatores explicam $80,65 \%$ da variância total da nuvem de dados, o que é satisfatório pelo critério da porcentagem da variância.

Tabela 1. Resultados dos autovalores para a extração de fatores componentes e variância total explicada pelos fatores.

\begin{tabular}{c|cccc|c|c|c}
\hline \multirow{2}{*}{ Componentes } & \multicolumn{2}{|c|}{ Autovalores $(\lambda)$ e variâncias iniciais } & \multicolumn{3}{|c|}{ Variâncias após rotação } \\
\cline { 2 - 7 } & $\begin{array}{c}\text { Variância } \\
\text { Total }\end{array}$ & $\begin{array}{c}\% \\
\text { variância }\end{array}$ & $\begin{array}{c}\text { Variância } \\
\text { acumulada }\end{array}$ & Total & $\begin{array}{c}\% \\
\text { variância }\end{array}$ & $\begin{array}{c}\text { Variância } \\
\text { Acumulada }\end{array}$ \\
\hline $\mathbf{1}$ & $\mathbf{4 , 5 8 4}$ & $\mathbf{5 0 , 9 2 8}$ & $\mathbf{5 0 , 9 2 8}$ & $\mathbf{4 , 3 3 1}$ & $\mathbf{4 8 , 1 2 6}$ & $\mathbf{4 8 , 1 2 6}$ \\
$\mathbf{2}$ & $\mathbf{1 , 5 6 7}$ & $\mathbf{1 7 , 4 0 9}$ & $\mathbf{6 8 , 3 3 7}$ & $\mathbf{1 , 7 3 2}$ & $\mathbf{1 9 , 2 4 9}$ & $\mathbf{6 7 , 3 7 6}$ \\
$\mathbf{3}$ & $\mathbf{1 , 1 0 8}$ & $\mathbf{1 2 , 3 1 4}$ & $\mathbf{8 0 , 6 5 2}$ & $\mathbf{1 , 1 9 5}$ & $\mathbf{1 3 , 2 7 6}$ & $\mathbf{8 0 , 6 5 2}$ \\
$\mathbf{4}$ & 0,687 & 7,638 & 88,289 & & & \\
$\mathbf{5}$ & 0,528 & 5,862 & 94,151 & & & \\
$\mathbf{6}$ & 0,329 & 3,650 & 97,802 & & & \\
7 & 0,175 & 1,949 & 99,751 & & & \\
8 & 0,017 & 0,184 & 99,935 & & & \\
9 & 0,006 & 0,065 & 100,00 & & & \\
\hline
\end{tabular}

Fonte: Resultados da pesquisa.

Na Tabela 2, as três primeiras colunas são os resultados para os três fatores extraídos, ou seja, as cargas fatoriais para cada variável em cada fator. A quarta coluna fornece a estatística, detalhando o grau em que cada variável é "explicada” pelas três componentes, denominada de comunalidade. Das duas últimas linhas, a primeira é a soma da coluna de cargas fatoriais ao quadrado (autovalores) e indica a importância relativa de cada fator na explicação da variância associada ao conjunto de variáveis analisado. A soma dos três fatores são 4,331, 1,732 e 1,195, respectivamente. Como esperado, a solução fatorial extraiu os fatores na ordem de sua importância, com o fator 1 explicando a maior parcela da variância $(48,13 \%)$, o fator 2 explicando $19,25 \%$ e o fator 3 
explicando $13,27 \%$ ). O número 7,258 representa a soma total de cargas fatoriais ao quadrado e indica a parcela total de variância extraída pela solução fatorial rotacionada.

A parcela total da variância explicada pela solução fatorial $(7,258)$ pode ser comparada com a variação total do conjunto de variáveis que é representada pelo traço da matriz fatorial. O traço é a variância total a ser explicada, obtida pela soma dos autovalores do conjunto de variáveis (soma da primeira coluna da Tabela 1), que é igual a 9,0, dado que cada variável tem um autovalor possível igual a 1,0. A soma total dos porcentuais de traço extraído para a solução fatorial, serve como índice para determinar o grau de adequação da solução fatorial em relação ao que todas as variáveis representam. $\mathrm{O}$ índice para esta solução mostra que 80,652 \% da variância total são representados pela informação contida na matriz fatorial da solução em termos dos três fatores. O índice é considerado alto, e as variáveis estão, como esperado, estreitamente relacionadas umas com as outras.

A soma em linha de cargas fatoriais ao quadrado gera a comunalidade, última coluna da Tabela 2. Estes números mostram a magnitude da variância em uma variável que é explicada pelos três fatores tomados juntos. O tamanho da comunalidade é um índice útil para avaliar o quanto de variância em uma dada variável é explicado pela solução fatorial. Comunalidades grandes indicam que uma grande parcela da variância em uma variável foi extraída pela solução fatorial. Uma comunalidade pequena, inferior a 0,50 , mostra que uma boa parte da variância contida em uma variável não é explicada pelos fatores.

Sempre que uma solução fatorial satisfatória é obtida, é importante atribuir um significado a ela. O processo envolve substantiva interpretação do padrão das cargas fatoriais para as variáveis, incluindo seus sinais, como esforço para nomear cada fator. Em geral, todas as cargas fatoriais significantes foram utilizadas no processo de interpretação, porém, as variáveis com maior carga influenciaram mais na seleção de nomes ou rótulo para representar os fatores.

A seleção das variáveis significativas que devem fazer parte de um fator é eleita com base na magnitude da carga fatorial (Tabela 2). Assim, as variáveis significativas podem ser eleitas, olhando-se da esquerda para a direita ao longo de cada linha e selecionando-se as cargas de 
maior valor. Adotando este processo, o fator 1 tem cinco cargas significativas, o fator 2 tem três cargas significativas e o fator 3, uma.

No primeiro fator estão as seguintes variáveis: Produção, Receita, Custo, Emprego e Concentração de mercado, ambas com sinais positivos e valores altos, demonstrando que todas variam juntas, estando coerente com as forças definidoras da estrutura e desempenho empresarial. Assim, uma maior quantidade produzida está associada a uma maior receita, dados os preços, a um custo total mais elevado e exige maior número de empregados, ceteris paribus. Para que a posição competitiva das empresas aumente, de acordo com os determinantes do fator 1, as estratégias dever ser ajustadas pata atenuar as ameaças das empresas rivais, de produtos substitutos e de novos entrantes no mercado. As variáveis vinculadas ao fator 1, em geral, estão fortemente atreladas às empresas de maior porte, com maior capacidade de exercer poder de mercado e conquistar maior parcela de mercado. Este fator pode ser chamado de dimensão competitividade tradicional, uma vez que os fatores influenciados por essas variáveis dizem respeito à obtenção de economias de escala e barreira à entrada (volume de vendas, número de empregos, poder de mercado, baixo custo médio) que se enquadram nas dimensões estrutura e desempenho do modelo ECD ou modelo tradicional, nas dimensões micro do modelo sistêmico e na força rivalidade e ameaça de entrantes do modelo de Porter.

No fator 2, foram incluídas as variáveis: Assistência técnica, Tecnologia de produção e Acesso a crédito. Estas variáveis estão relacionadas às empresas de diversos tamanhos, evidenciando a capacidade de criar vantagem competitiva por meio da qualidade total dos produtos, administração de recursos financiados e obtenção de apoio técnico especializado. A combinação dessas variáveis exige o desenho de estratégias competitivas e a articulação com instituições financeiras e de assistência técnica, podendo representar a dimensão gestão competitiva. Esta dimensão de conduta está ligada às forças competitivas do modelo tradicional, das cinco forças de Porter e da competitividade sistêmica, cujos efeitos se manifestam ex-post na forma de desempenho competitivo.

O terceiro fator foi composto por apenas a variável Diversificação da produção. Este fator está associado apenas às empresas multiprodutos e foi evidenciada nas micro, pequenas e médias empresas pesquisadas. 
As empresas multiprodutos fabricam vários tipos de polpa de frutas e conseguem reduzir risco de mercado por se adequar à dinâmica do varejo e das empresas de distribuição. Esta é uma força importante para as empresas vencerem concorrência e ampliarem a participação no mercado, diante da nova face da arena competitiva das empresas. Ela pode representar a dimensão competitividade sustentável das empresas de polpa de frutas.

Tabela 2. Matriz de cargas fatoriais ( $\alpha$ ) após a rotação ortogonal pelo método Varimax.

\begin{tabular}{l|c|c|c|c}
\hline \multirow{2}{*}{ Variáveis } & \multicolumn{3}{|c|}{ Fatores } & Comunali- \\
\cline { 2 - 4 } & $\mathrm{F} 1$ & $\mathrm{~F} 2$ & $\mathrm{~F} 3$ & dade \\
\hline Quantidade produzida & $\mathbf{0 , 9 7 7 0}$ & 0,1114 & $-0,0362$ & 0,9682 \\
Receita total & $\mathbf{0 , 9 7 2 4}$ & 0,1661 & 0,0727 & 0,9783 \\
Custo total de produção & $\mathbf{0 , 8 9 6 8}$ & 0,1210 & $-0,1513$ & 0,8418 \\
Concentração de mercado & $\mathbf{0 , 9 0 9 9}$ & 0,0325 & 0,0996 & 0,8389 \\
Número de emprego & $\mathbf{0 , 8 5 2 7}$ & 0,1686 & 0,2718 & 0,8295 \\
Assistência Técnica & 0,0937 & $\mathbf{0 , 8 0 9 7}$ & 0,0026 & 0,6644 \\
Tecnologia de produção & 0,2032 & $\mathbf{0 , 6 5 2 2}$ & $-0,4322$ & 0,6534 \\
Acesso a crédito & 0,0714 & $\mathbf{0 , 7 5 2 4}$ & 0,1478 & 0,5930 \\
Diversificação da produção & 0,1300 & 0,0360 & $\mathbf{0 , 9 3 4 3}$ & 0,8911 \\
\hline Soma de quadrado do autovalor & 4,331 & 1,732 & 1,195 & 7,258 \\
Porcentual do traço (\%) & 48,125 & 19,249 & 13,276 & 80,652 \\
\hline
\end{tabular}

Fonte: Resultados da pesquisa.

\subsection{Escores fatoriais e índice de desempenho competitivo}

Os escores fatoriais associados a cada uma dessas dimensões foram determinados para estimar o IDC. Os pesos-betas utilizados na estimativa dos escores fatoriais estão na Tabela 3. Substituindo-se estes pesosbetas na equação 4, que utiliza as variáveis normalizadas, obtêm-se os escores fatoriais originais.

Os escores fatoriais assim determinados permitem que sejam visualizadas as direções da influência de cada dimensão fatorial sobre o IDC. Na Tabela 3, os pesos-betas associados a um fator respectivo são positivos e os vinculados aos demais fatores apresentam sinais negativos e 
positivos. Porém, a característica fundamental é que os maiores pesos se vinculam aos fatores respectivos.

Tabela 3. Matriz de coeficientes de regressão ou pesos-betas dos escores fatoriais.

\begin{tabular}{l|c|c|c}
\hline \multirow{2}{*}{\multicolumn{1}{c|}{ Variáveis }} & \multicolumn{3}{|c}{ Fatores } \\
\cline { 2 - 4 } & $\mathrm{F} 1$ & $\mathrm{~F} 2$ & $\mathrm{~F} 3$ \\
\hline Quantidade produzida $\left(\mathrm{w}_{1}\right)$ & 0,2414 & $-0,0547$ & $-0,0886$ \\
Receita total $\left(\mathrm{w}_{2}\right)$ & 0,2259 & $-0,0104$ & 0,0097 \\
Custo total de produção $\left(\mathrm{w}_{3}\right)$ & 0,2272 & $-0,0476$ & $-0,1811$ \\
Concentração de mercado $\left(\mathrm{w}_{4}\right)$ & 0,2247 & $-0,0860$ & 0,0263 \\
Número de emprego $\left(\mathrm{w}_{5}\right)$ & 0,1811 & 0,0222 & 0,1889 \\
Assistência Técnica $\left(\mathrm{w}_{6}\right)$ & $-0,0781$ & 0,5076 & 0,0604 \\
Tecnologia de produção $\left(\mathrm{w}_{7}\right)$ & $-0,0003$ & 0,3581 & $-0,3328$ \\
Acesso a crédito $\left(\mathrm{w}_{8}\right)$ & $-0,0865$ & 0,4853 & 0,1820 \\
Diversificação da produção $\left(\mathrm{w}_{9}\right)$ & $-0,0343$ & 0,0812 & 0,7961 \\
\hline
\end{tabular}

Fonte: Resultados da pesquisa.

Os escores fatoriais estimados foram apresentados na Tabela 4. Fazendo-se a padronização desses escores originais, obtêm-se os escores fatoriais padronizados, com base nos quais se determina o IDC, mediante emprego da equação 8, que tem a finalidade de hierarquizar as empresas de acordo com seu desempenho competitivo.

Na Tabela 4, os três escores fatoriais originais são positivos para as duas primeiras empresas e dois positivos para as duas empresas seguintes. Um sinal positivo indica que a empresa está atuando de tal forma que as variáveis definidoras das dimensões fatoriais apresentam influência positiva para o desempenho competitivo e um sinal negativo significa que os efeitos das forças positivas superam os efeitos das forças negativas.

As duas primeiras empresas da Tabela 4 estão obtendo resultado positivo na gestão das variáveis definidoras dos três escores fatoriais. Isto significa um tamanho da instalação adequada, participação de mercado e custo baixo de produção para o fator 1; utilização de assistência técnica, acesso a crédito e uso de tecnologia, para o fator 2; produção diversificada, ao fabricar vários tipos de polpa de frutas, para o fator 3. A terceira empresa, por sua vez, apresentou um sinal negativo para o 
fator 3, por ser uma empresa uniproduto. A quarta empresa apresentou sinal negativo para o fator 2 , em função do não acesso à assistência técnica especializada e tecnologia avançada, principalmente.

Tabela 4. Valores dos escores fatoriais originais e padronizados e o índice de desempenho competitivo.

\begin{tabular}{|c|c|c|c|c|c|c|c|}
\hline \multirow{2}{*}{ Empresa } & \multicolumn{3}{|c|}{ Escore fatorial original } & \multicolumn{3}{|c|}{ Escore fatorial padronizado } & \multirow{2}{*}{ IDC } \\
\hline & Fator 1 & Fator 2 & Fator 3 & FP1 & FP2 & FP3 & \\
\hline Média empresa 1 & 4,439 & 0,545 & 0,870 & 1,000 & 0,608 & 0,733 & 0,863 \\
\hline Pequena empresa 1 & 0,263 & 1,230 & 0,668 & 0,207 & 0,811 & 0,672 & 0,428 \\
\hline Pequena empresa 2 & 0,923 & 1,013 & $-0,873$ & 0,332 & 0,747 & 0,211 & 0,411 \\
\hline Média empresa 2 & 0,142 & $-0,227$ & 1,764 & 0,184 & 0,380 & 1,000 & 0,365 \\
\hline Microempresa 1 & $-0,680$ & 0,764 & 1,546 & 0,028 & 0,673 & 0,935 & 0,331 \\
\hline Pequena empresa 3 & $-0,827$ & 0,789 & 1,602 & 0,000 & 0,681 & 0,951 & 0,319 \\
\hline Pequena empresa 4 & $-0,383$ & 0,391 & 0,938 & 0,084 & 0,563 & 0,753 & 0,309 \\
\hline Pequena empresa 5 & $-0,138$ & 1,154 & $-0,757$ & 0,131 & 0,789 & 0,246 & 0,307 \\
\hline Pequena empresa 6 & 0,087 & 0,475 & $-0,358$ & 0,174 & 0,588 & 0,365 & 0,304 \\
\hline Pequena empresa 7 & $-0,388$ & 1,185 & $-0,728$ & 0,083 & 0,798 & 0,255 & 0,282 \\
\hline Microempresa 2 & $-0,480$ & $-0,245$ & 1,388 & 0,066 & 0,374 & 0,888 & 0,275 \\
\hline Microempresa 3 & $-0,351$ & $-0,271$ & 1,116 & 0,091 & 0,367 & 0,806 & 0,274 \\
\hline Microempresa 4 & 0,538 & $-0,590$ & $-0,650$ & 0,259 & 0,272 & 0,278 & 0,265 \\
\hline Microempresa 5 & $-0,809$ & 1,867 & $-1,176$ & 0,003 & 1,000 & 0,121 & 0,261 \\
\hline Microempresa 6 & $-0,454$ & 0,560 & $-0,190$ & 0,071 & 0,613 & 0,416 & 0,257 \\
\hline Microempresa 7 & $-0,737$ & 1,226 & $-0,669$ & 0,017 & 0,810 & 0,273 & 0,249 \\
\hline Pequena empresa 8 & 0,087 & $-1,296$ & 0,977 & 0,174 & 0,063 & 0,765 & 0,245 \\
\hline Pequena empresa 9 & $-0,088$ & 0,181 & $-1,017$ & 0,140 & 0,501 & 0,169 & 0,231 \\
\hline Microempresa 8 & $-0,089$ & 0,145 & $-0,987$ & 0,140 & 0,490 & 0,177 & 0,230 \\
\hline Pequena empresa 10 & 0,866 & $-1,015$ & $-1,581$ & 0,322 & 0,146 & 0,000 & 0,227 \\
\hline Microempresa 9 & 0,013 & $-0,508$ & $-0,425$ & 0,160 & 0,297 & 0,346 & 0,223 \\
\hline Pequena empresa 11 & $-0,528$ & $-0,377$ & $-0,117$ & 0,057 & 0,335 & 0,438 & 0,186 \\
\hline Microempresa 10 & $-0,547$ & $-1,218$ & 1,085 & 0,053 & 0,086 & 0,797 & 0,183 \\
\hline Microempresa 11 & 0,199 & $-1,509$ & $-0,842$ & 0,195 & 0,000 & 0,221 & 0,153 \\
\hline Microempresa 12 & $-0,146$ & $-1,481$ & $-0,432$ & 0,129 & 0,008 & 0,343 & 0,136 \\
\hline Microempresa 13 & $-0,409$ & $-1,404$ & $-0,604$ & 0,079 & 0,031 & 0,292 & 0,103 \\
\hline Microempresa 14 & $-0,504$ & $-1,384$ & $-0,550$ & 0,061 & 0,037 & 0,308 & 0,096 \\
\hline Valor máximo & 4,439 & 1,867 & 1,764 & & & Média & 0,278 \\
\hline Valor mínimo & $-0,827$ & $-1,509$ & $-1,581$ & & & Mediana & 0,261 \\
\hline Razão da variância & 0,597 & 0,239 & 0,165 & & & & \\
\hline
\end{tabular}


Um grupo de seis empresas apresentou sinal negativo apenas para o fator 1, o que revela inadequação da escala de produção e, por conseqüência, custos elevados e dificuldade com as variáveis acesso a crédito e ao mercado. Um outro grupo de 13 empresas apresentou sinal negativo em dois fatores. Por fim, um grupo crítico de quatro empresas apresentou sinal negativo para os três fatores. Esta estrutura de debilidade caracteriza a posição da empresa no ranking gerado pelo IDC.

Estes resultados permitem posicionar individualmente as empresas com respeito às suas fragilidades e pontos fortes, inclusive com a magnitude do impacto, segundo a dimensão fatorial. Constitui, portanto, um passo fundamental para orientar as estratégias empresariais no nível microeconômico de análise, bem como as ações de política pública que se definem no nível mesoeconômico.

Ordenando-se os valores do IDC, obteve-se uma empresa com alto grau de desempenho competitivo, vez que o IDC > 0,70 (a média empresa 1 obteve IDC $=0,863$ ). Três empresas apresentaram grau de desempenho competitivo intermediário, com IDC entre 0,35 e 0,70 (pequena empresa $1 \mathrm{com}$ IDC $=0,428$, pequena empresa 2 com IDC $=0,411$, média empresa 2 com IDC $=0,365$ ) e as demais 23 empresas apresentam baixo grau de competitividade. Os resultados revelaram que as quatro empresas com IDC de médio a superior foram as que ajustaram de forma mais eficiente suas estratégias competitivas.

Se forem considerados os valores médios e medianos do IDC para efeito de análise, tem-se que 10 empresas estariam com IDC acima do índice médio e 14 empresas estariam acima do índice mediano. Este resultado pode servir como orientador para as empresas adotarem medidas que as conduzam a evoluir, atuando no conjunto de variáveis que definem suas dimensões competitivas.

O conjunto de empresas com IDC abaixo da média está retardando a construção de vantagens competitivas sustentáveis e aumentando a ameaça de entrada de novos concorrentes no mercado. Este fato exige atenção para que este grupo de empresas não comprometa as oportunidades das empresas que estão evoluindo mediante criação de vantagens competitivas. 


\subsection{Análise econométrica}

Os resultados da análise de regressão revelaram presença de heterocedasticidade, o que foi corrigido pelo método de White. Não foram observados problemas de multicolinearidade e de autocorrelação. As variáveis incluídas na regressão explicam $65,3 \%$ das variações no IDC entre as empresas. A estatística $\mathrm{F}=10,32$ foi significativa a $1 \%$ de probabilidade. Esses resultados atestam o emprego do modelo de regressão (Tabela 5).

Tabela 5. Estimativas dos coeficientes de regressão múltipla do IDC das empresas de polpa de frutas.

\begin{tabular}{lccccc}
\hline Variável & Coeficiente & Desvio padrão & Estatística t & Probabilidade \\
\hline Constante & 0,1804 & 0,0451 & 4,0054 & 0,0006 \\
Fornecedor & 0,0004 & 0,0001 & 3,0000 & 0,0068 \\
Margem de lucro & 0,0055 & 0,0023 & 2,3868 & 0,0260 \\
Capacidade ociosa & $-0,0014$ & 0,0010 & $-1,4563$ & 0,1595 \\
Organização em departamento & 0,0524 & 0,0392 & 1,3366 & 0,1950 \\
\hline R-quadrado & 0,6523 & Média da var. dependente & 0,2782 \\
R-quadrado ajustado & 0,5891 & Desvio padrão var. dependente & 0,1423 \\
Desvio padrão da regressão & 0,0912 & Estatística - F & 10,318 \\
Estatística Durbin-Watson - d & 1,9340 & Probabilidade & 0,00007 \\
\hline
\end{tabular}

Fonte: Resultados da pesquisa.

As variáveis - Fornecedores e Margem de Lucro - apresentaram sinais significativos e coerentes com o esperado, mostrando que variações percentuais unitárias nestas variáveis produzem mudanças no IDC de $0,027 \%$ ( $=0,0004 \times$ Fmédio) e 0,0055\%, respectivamente, na mesma direção. Isto significa que mudanças nessas variáveis podem alterar a posição das empresas no ranking do desempenho competitivo. As duas outras variáveis, não obstante terem apresentado sinais corretos, não foram significativas.

Em geral, esses resultados mostram que a ligação das empresas com fornecedores é forte determinante do desempenho competitivo das empresas, referendando os postulados teóricos da economia industrial de que o poder dos fornecedores afeta a rentabilidade das empresas. Da mesma forma, a margem de lucro, tida como um indicador clássico de 
desempenho empresarial, também se mostrou significativo para as empresas de polpa de frutas paraenses.

A variável capacidade ociosa não está influenciando o desempenho competitivo das empresas, sobretudo porque os altos níveis retratam os problemas de sazonalidade na produção de matéria-prima, que afeta por igual a maioria das empresas. A organização da empresa em departamentos, embora contribua para aumentar a racionalidade e eficiência do processo produtivo, está sendo experimentado em um número reduzido de empresas e sem evidência nos resultados.

\section{Considerações finais}

O modelo de análise fatorial ajustou-se bem às variáveis indicadoras do desempenho competitivo das empresas da indústria de polpa de frutas paraense, de acordo com a significância dos testes estatísticos utilizados.

Os resultados permitiram a extração de três fatores, representativos das dimensões que determinam a competitividade empresarial: Competitividade tradicional, explicando $48,13 \%$ da variância total e 59,67 da variância comum; Gestão competitiva, respondendo por 19,25\% da variância total e 23,87 da variância comum; e Competitividade sustentável, que explicou $13,27 \%$ da variância total e 16,46\% da variância comum. Essas dimensões permitiram estimar os escores fatoriais utilizados na construção do índice de desempenho competitivo (IDC).

O IDC permitiu posicionar as empresas conforme o grau de competitividade na indústria de polpa de frutas. Uma empresa apresentou alto nível de desempenho competitivo, revelando efeitos positivos de suas estratégias nas três dimensões fatoriais identificadas. Três empresas apresentaram desempenho competitivo intermediário, em que duas revelaram debilidade em uma das três dimensões fatoriais. As demais empresas apresentaram baixo desempenho competitivo.

Em relação à média, 10 empresas foram enquadradas acima do IDC médio e 14 empresas apresentaram IDC acima da mediana. O conjunto de empresas com IDC abaixo da mediana apresentou debilidade em pelo menos duas das três dimensões fatoriais.

A análise de regressão revelou que as variáveis Margem de lucro e Fornecedores apresentam associação positiva e significativa com o IDC 
e as variáveis capacidade ociosa e organização em departamentos não exerceram influência no IDC.

O IDC permitiu identificar o grau do desempenho competitivo de cada empresa e a dimensão fatorial em que apresentou fragilidade, mostrando-se adequado para orientar a formulação de estratégias competitivas no âmbito das empresas, bem como para o desenho e aplicação dos instrumentos de política pública.

\section{Referências bilbiográficas}

BAIN, J.S. Industrial organization. 2 ed. New York: Wiley, 1968.

BRAGA, H.C. Estrutura de Mercado e desempenho da indústria brasileira: 1973/75. Rio de Janeiro: FGV, 1980. (Tese, 06).

COUTINHO, L., FERRAZ, J.C. (coord.) Estudo da competitividade da indústria brasileira. 2 ed. Campinas: Papirus, 1994.

DILLON, W.R.; GOLDSTEIN, M. Multivariate analysis: methods and applications. New York: John Wiley \& Sons, 1984.

ESSER, K.; HILLEBRAND, W.; MESSNER, D.; MEYER-STANNER, J. Systemic competitiveness - new challenges to business and politics. Economics, v.59, 1999.

FAIRBANKS, M., LINDSAY, S. Arando o mar: fortalecendo as fontes ocultas do crescimento em países em desenvolvimento. Rio de Janeiro: Qualitymark Ed., 2002.

FARINA, E.M.M.Q., ZILBERSZTAJN, D. (Coord.) Competitividade no agribusiness brasileiro. São Paulo: PENSA?FIA/FEA?USP, 1998.

FERRAZ, J.C.; KUPFER, D., HAGUENAUER, L. Made in Brasil: desafios competitivos para a indústria. Rio de Janeiro: Campus, 1995.

HAIR JR, J.F., ANDERSON, R.E., TATHAM, R.L., BLACK, W.C. Análise multivariada de dados. 5. ed. Porto Alegre: Bookman, 2005.

JOHNSON, R.A.; WICHERN, D.W. Applied multivariate statistical analysis. Prentice-Hall, 1992.

KITABAYASHI, R.T. Estratégia competitiva do varejo supermercadista 
da Região Metropolitana de Belém: 1990 a 2004. 2005. 165 f. Dissertação (Mestrado em Economia) - Universidade da Amazônia.

KUPFER, D., HASENCLEVER, L. (Orgs.) Economia industrial: fundamentos teóricos e práticas no Brasil. Rio de Janeiro: Campus, 2002.

MINGOTI, S.A. Análise de dados através de métodos de estatística multivariada: uma abordagem aplicada. Belo Horizonte: Editora UFMG, 2005.

OCDE. Technology and the economy: the key relationships. Paris: OCDE, 1992.

PORTER, M.E. The competitive advantage of nations. New York: Free Press, 1990.

PORTER, M.E. Como as forças competitivas moldam a estratégia. In: PORTER, M.E. Competição = on competition: estratégias competitivas essenciais. Rio de Janeiro: Campus, 1999. p.27-45.

POSSAS, M.L. Estrutura de mercado em oligopólio. 2 ed. São Paulo: Hucitec, 1987.

POSSAS, M.L. Competitividade: fatores sistêmicos e política industrial - implicações para o Brasil. In: Castro, A.B. (org.), Possas, M.L. (org.), Proença, A. (org.) Estratégias empresariais na indústria brasileira: discutindo mudanças. Rio de Janeiro: Forense, 1996. P.71-117.

REDESIST. Rede de Pesquisa em Sistemas Produtivos e Inovativos Locais. Rede de pesquisa interdisciplinar, sediada no Instituto de Economia da Universidade Federal do Rio de Janeiro. Disponível em: < http:// www.ie.ufrj.br/redesist/ > . Acesso em 10 jan. 2004.

REIS, E. Estatística multivariada aplicada. 2. ed. Lisboa: Silabo, 2001.

SANTANA, A.C. de. Análise da competitividade sistêmica da indústria de madeira no Estado do Pará. Revista de Economia e Agronegócio, Viçosa - MG, v. 1, n. 2, p. 205-230, 2003.

SANTANA, A.C. de. Análise do desempenho competitivo das agroindústrias de polpa de frutas do Estado do Pará. Revista de Economia e Agronegócio, Viçosa - MG, v. 2, n. 4, p. 495-523, 2004. 
SANTANA, A.C. de. Elementos de economia, agronegócio e desenvolvimento local. Belém: GTZ; TUD; UFRA, 2005. p.133-142. (Série Acadêmica, 01).

SANTANA, A.C. de. Métodos quantitativos em economia: elementos e aplicações. Belém: UFRA, 2003.

SANTANA, A.C. de. A competitividade sistêmica das empresas de madeira da Região Norte. Belém: UFRA, 2002.

SANTANA, A.C. de; CARVALHO, D.F.; MENDES, F.A.T.M.. O desempenho competitivo das empresas de polpa de frutas do APL de fruticultura do nordeste paraense. In: CONGRESSO BRASILEIRO DE ECONOMIA E SOCIOLOGIA RURAL, 43, Ribeirão Preto, 2005. Anais. Brasília: SOBER, 2005.

SANTANA, A.C. de. A indústria de madeira do Estado do Pará: análise de competitividade. Novos Cadernos Naea, Belém-PA, v. 4, n. 2, p. 83$114,2001$.

SANTANA, A.C. de, CARVALHO, D.F., MENDES, F.A.T. Organização, mercado e competitividade das empresas de polpa de frutas do Estado do Pará. Belém: FIDESA/UNAMA, 2006. (Relatório Final da Pesquisa).

SHY, O. Industrial organization: theory and applications. London: MIT Press, 1997.

SILVA, M.C.N. Competitividade das agroindústrias de polpa de frutas das mesorregiões metropolitana de Belém e nordeste paraense (1996 a 2003). 2006. 130 f. Dissertação (Mestrado em Economia) - Universidade da Amazônia.

SPSS. SPSS Base 7.5 applications guide. Chicago: SPSS Inc., 1997.

TIROLE, J. The theory of industrial organization. Cambridge: MIT Press, 2001.

Recebido em março de 2006 e revisto em março de 2007. 